\title{
Two unusual organisms, Aspergillus terreus and Metschnikowia pulcherrima, associated with the lung disease of ankylosing spondylitis
}

\author{
W. P. U. KENNED Y ${ }^{1}, L$. J . R. MILNE ${ }^{2}, W \cdot B L Y T H^{3}$, a nd \\ G. K. CROMPTON ${ }^{1}$ \\ Respiratory Diseases Unit, Northern General Hospital, Edinburgh and Department of Respiratory \\ Diseases, University of Edinburgh ${ }^{1}$, Central Microbiological Laboratory, Western General Hospital, \\ Edinburgh ${ }^{2}$, and Department of Botany, University of Edinburgh ${ }^{3}$
}

\begin{abstract}
Two male patients with ankylosing spondylitis and upper lobe fibrosis and cavitation are described. A pneumonic disease in one was associated with mycological and serological evidence of infection with Aspergillus terreus but no other aspergillus species. A large pulmonary mycetoma developed in the second patient and among a number of other fungal isolates was found the yeast Metschnikowia pulcherrima. The association of ankylosing spondylitis with bronchopulmonary aspergillosis is considered; $\boldsymbol{A}$. terreus is described for the first time as a human pulmonary pathogen, and the possible pathogenicity of $M$. pulcherrima in the debilitated human subject is discussed.
\end{abstract}

Aspergillus fumigatus is the fungal organism most commonly productive of pulmonary disease in this country, but a number of other Aspergillus species have, from time to time, been recognized as pathogenic to man. Aspergillus terreus, however, has infrequently been recorded in human disease and seems rarely, if at all, to have been suspected as a respiratory pathogen. Nor, to our knowledge, has the yeast Metschnikowia pulcherrima (previously Candida pulcherrima) been implicated in human pulmonary infection.

Two patients are described, each with longstanding ankylosing spondylitis. Pulmonary changes were present in both, characterized by fibrosis and cavitation in the upper lobes. The first patient showed evidence of infection with $A$. terreus. The second developed an $A$. fumigatus mycetoma, and $M$. pulcherrima was isolated from a number of sources.

\section{CASE REPORTS}

PATIENT 1 A 54-year-old man was referred because of recent haemoptysis associated with an abnormality on the chest radiograph. He was moderately crippled by ankylosing spondylitis which had been diagnosed at the age of 20 . At 26 he received a

Requests for reprints should be sent to Dr. G. K. Crompton course of deep $x$-ray therapy to the spine. He smoked 15 to 20 cigarettes daily and had a morning cough but no breathlessness or wheeze. For four months the cough had been productive of white or yellowish sputum, and two weeks before presentation he noticed a few small spots of blood which recurred on one occasion several days later.

$\mathrm{He}$ had a moderate kyphosis and flexion deformity of both hips. Finger clubbing was not present. The chest radiograph showed an opacity in the upper zone of the left lung field and elevation of the left pulmonary hilar shadow. The forced expiratory volume in 1 second was 2.0 litres and the forced vital capacity 2.25 litres. A Heaf test was grade I positive, and three specimens of sputum were negative on direct examination and on culture for Mycobacterium tuberculosis.

He remained well, without treatment, for three months but then developed symptoms of a respiratory infection with a productive cough and rightsided pleural pain. His family doctor prescribed ampicillin but he continued to feel unwell and after three weeks attended the outpatient department. Coarse crepitations were audible over the right lower lung posteriorly. The chest radiograph showed an opacity in the right lower zone and a smaller one in the left lower zone (Fig. 1). He was admitted to hospital for treatment and for investigation of the possibility of pulmonary aspergillosis. His haemoglobin was $11.6 \mathrm{~g} / 100 \mathrm{ml}$, white cell count $6,900 /$ $\mathrm{mm}^{3}$, and ESR $116 \mathrm{~mm}$ in the first hour. 


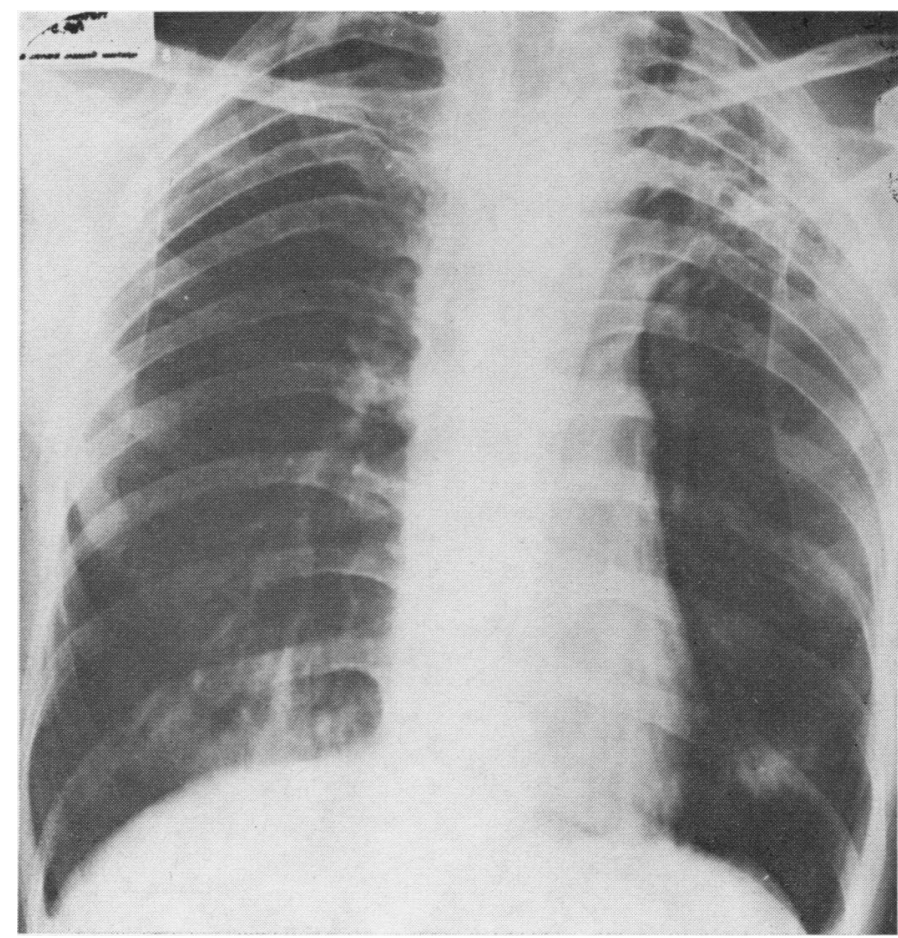

FIG. 1. Patient 1. Chest radiograph showing fibrosis and cavitation in the left upper zone and 'pneumonic' shadow in both lower zones.

During a 12-day period, 10 sputum specimens were specifically examined for fungi (see Methods) and in each specimen an aspergillus was isolated in moderate to large numbers, later identified as $A$. terreus. Bacteriological examination of the sputum yielded a commensal flora; examination and culture for tubercle bacilli were negative. Skin testing with $A$. fumigatus extract (Bencard) was negative. Serological studies are described below.

Clinical recovery was satisfactory, without antibiotic therapy, and the radiographic abnormalities in the lower zones cleared. $A$. terreus was still present in the sputum, however. Following discharge from hospital he was seen at monthly intervals and remained well. On each occasion the sputum was examined mycologically and yielded profuse growths of A. terreus. Six months after discharge skin tests were carried out by the prick method, using both proprietary solutions and an extract of broth culture fluid of the patient's own organism. Dermal sensitivity to $A$. terreus was demonstrated whereas there was no reaction to $A$. fumigatus (Table). He was most recently seen nine months after discharge when he was well and his sputum, for the first time, showed absence of fungal growth.

PATIENT 2 A 43-year-old man was admitted to hospital with a two-month history of malaise, anorexia,
T A B L E

PATIENT I: PRICK TESTING TO FUNGAL ALLERGENS

\begin{tabular}{l|l|l}
\hline \multicolumn{1}{c|}{ Solution } & Wheal & Grading \\
\hline Control & None & - \\
A. fumigatus $5 \%$ (Bencard) & None & - \\
A.terreus $1 \%$ (Bencard) & $2 \mathrm{~mm}$ & ++ \\
A.terreus $1 \%$ (patient's) & $3 \mathrm{~mm}$ & +++ \\
A. terreus $10 \%$ (patient's) & $6 \mathrm{~mm}$ & ++++ \\
\hline
\end{tabular}

weight loss, and cough productive of green sputum. $\mathrm{He}$ had a rigid spine due to ankylosing spondylitis dating from the age of 14 years. Between the ages of 20 and 26 he had received five separate courses of radiotherapy, varying in dose from 1,500 to 2,000 R. A chest radiograph showed a large thin-walled cavity, about $4 \mathrm{~cm}$ in diameter, occupying the right lung apex and containing in its lower part a dense rounded opacity (Fig. 2). Anteroposterior tomography indicated an intracavitary mycetoma. A radiograph of the cervical spine taken four years previously had shown no abnormalities in the lung apices. A Heaf test was grade I positive, and repeated examination and culture of the sputum for tubercle bacilli were negative. Mycelial fragments were demonstrated on microscopical examination of the sputum, and $A$. fumigatus was isolated on culture. Skin testing with $A$. fumigatus extract was 


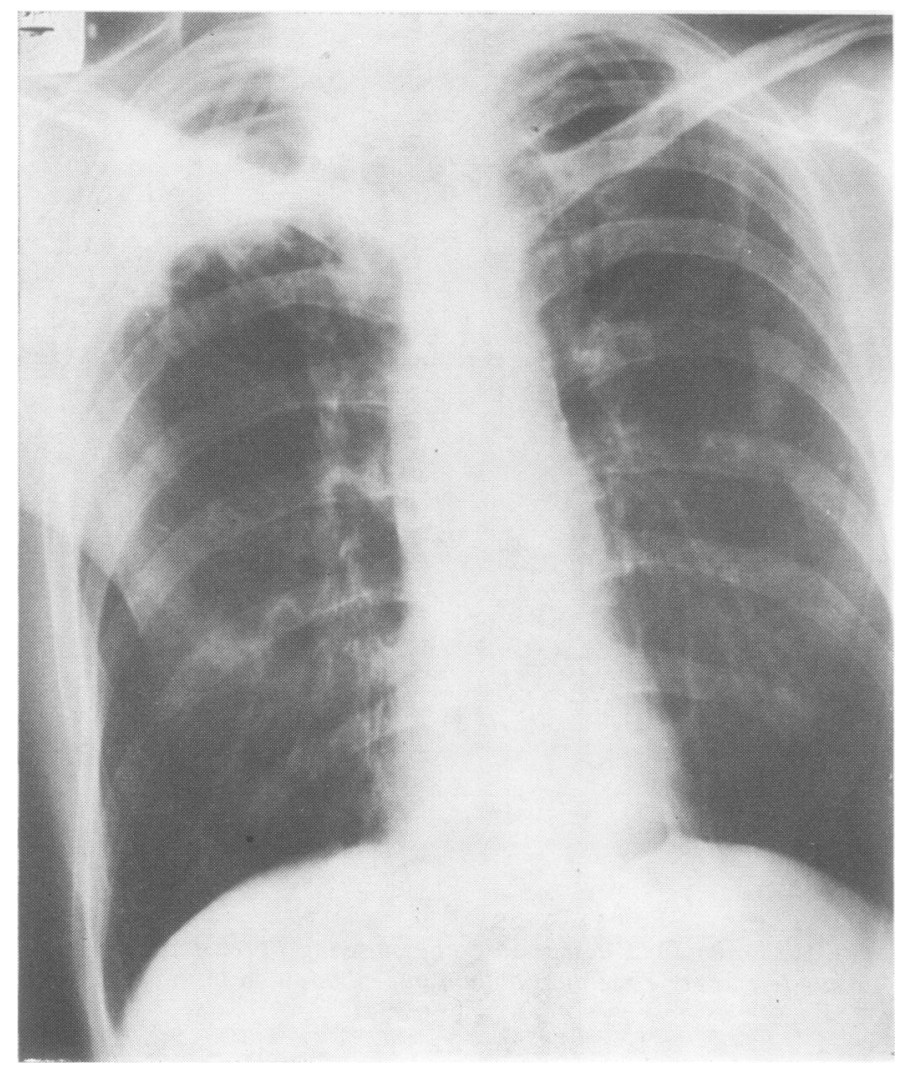

FIG. 2. Patient 2. Chest radiograph showing a mycetoma within a large right upper zone cavity.

negative, but precipitating antibodies against $A$. fumigatus were present in the serum. It was decided to keep the patient under observation without specific treatment, but after two visits to the clinic he failed to attend and was not seen again until readmission to hospital 22 months later. He was wasted, pale, and had marked finger clubbing. Radiographic examination of the chest showed a considerable increase in size of the right upper lobe cavity (Fig. 3). There also appeared to be cavitation in the left upper lobe. Serial sputum cultures were negative for Myco. tuberculosis, but $A$. fumigatus and other aspergillus species were cultured. A pink yeast, subsequently identified as Metschnikowia (Candida) pulcherrima, was isolated from sputum and urine.

Despite supportive therapy his condition deteriorated. He developed diarrhoea which did not respond to treatment with salicylazosulphapyridine. During the sixth week treatment with the synthetic antimycotic clotrimazole was started $(100 \mathrm{mg} / \mathrm{kg}$ body weight $/ 24$ hours). There appeared to be an initial improvement, but he then developed increasing diff- culty in breathing and he died on the tenth day after clotrimazole therapy had begun.

Necropsy findings Much of the right pleural sac was obliterated by old fibrous adhesions. The upper lobe of the right lung contained a cavity $12.5 \mathrm{~cm}$ in $D$ diameter within which was a solid spherical myce- 을. toma measuring $2.5 \mathrm{~cm}$ in diameter (Fig. 4). A N cavity of $6 \mathrm{~cm}$ diameter was present in the left $\sigma$ upper lobe. There were firm areas of consolidation $N$ in the right lower lobe, and elsewhere both lungs $N$ were congested and oedematous. The superior vena $\sigma$ cava was occluded by antemortem thrombus. The large bowel was affected by a form of chronic $\stackrel{0}{工}$ ulcerative colitis.

MYCOLOGICAL METHODS AND RESULTS (PATIENT 1) SPUTUM SMEAR Sputum samples were digested with pancreatin (Oxoid). The sediment obtained after $\mathbb{D}$ centrifugation was used to make smears which were stained with polychrome methylene blue. This 


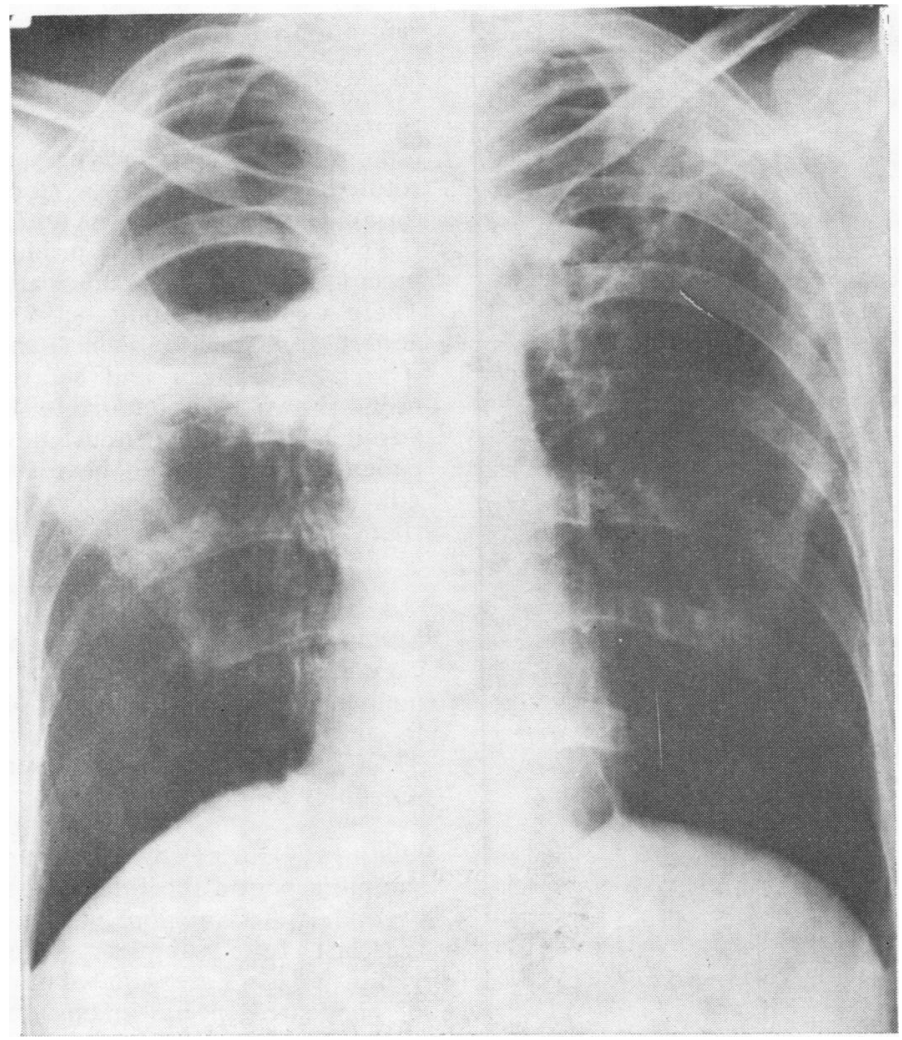

FIG. 3. Patient 2. Chest radiograph showing considerable increase in size of right upper lobe cavity and evidence of cavitation in left upper lobe.

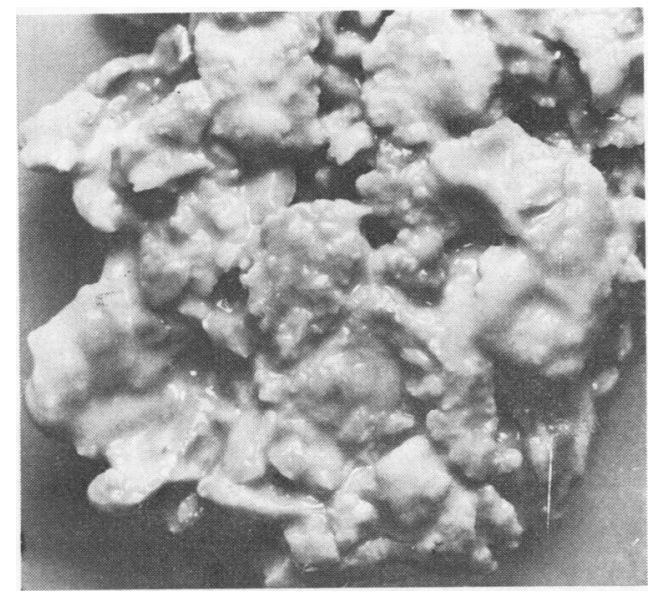

FIG. 4. Patient 2. The mycetoma removed at necropsy from the right upper lobe cavity. method was reported by Curry (1949) for the diagnosis of dermatophytes and has been found equally effective for staining hyphae in sputum.

SPUTUM CULTURE Two malt peptone agar plates were inoculated from each sputum. The entire sputum was spread over the surface of the medium of one plate with a cotton wool swab and incubated at $30^{\circ} \mathrm{C}$. This swab was then used to inoculate the second plate which was incubated at $37^{\circ} \mathrm{C}$.

SPUTUM SMEAR RESULTS The digested sputum was shown to contain branched septate hyphae which corresponded to the isolation of $A$. terreus on culture.

SPUTUM CULTURE RESULTS Daily specimens were received for 10 consecutive days and then at monthly intervals for six months following discharge from hospital. Culture consistently produced large numbers of $A$. terreus, a few colonies of which were also obtained on the swab plate (Fig. 5). No other filamentous fungi were isolated but occasionally small numbers of yeasts were recorded.

During the period of investigation it was observed 


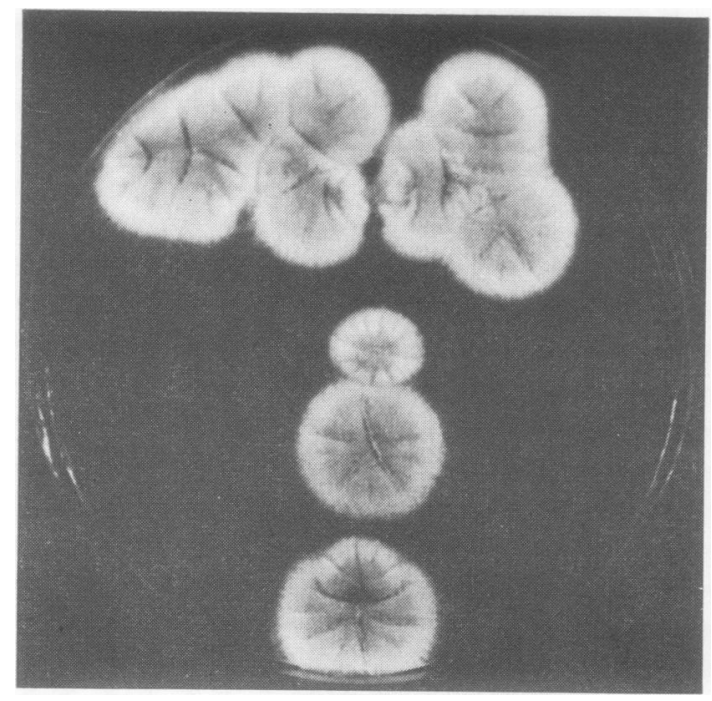

FIG. 5. Patient 1. Swab plate incubated for 48 hours showing colonies of A. terreus.

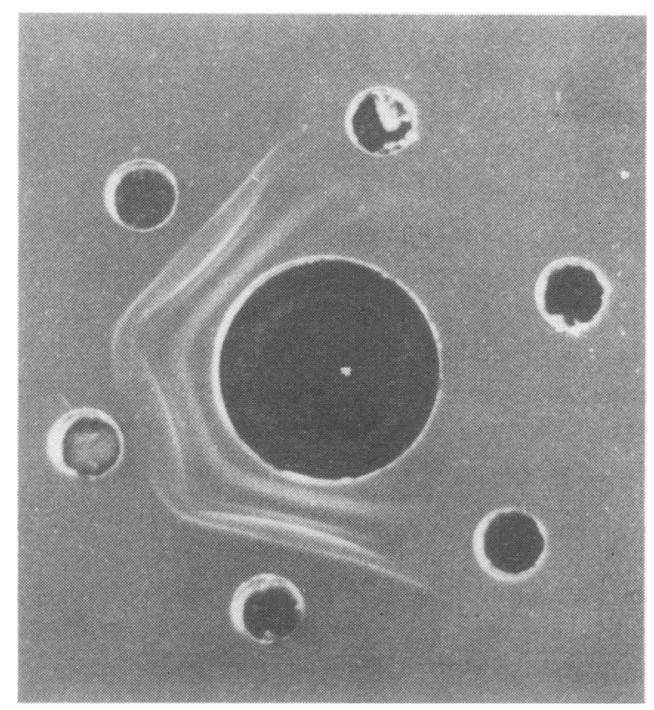

FIG. 6. Patient 1. Serial dilution of A. terreus antigen in gel diffusion against serum.

that considerable variation occurred in the isolates of $A$. terreus which were obtained. The significance of this variation is obscure.

\section{SEROLOGICAL METHODS AND RESULTS (PATIENT 1)}

A 3-week-old culture filtrate of the $A$. terreus isolated from this patient was dialysed against running tap water for three days and freeze-dried after con- centration with polyethylene glycol 6000 . The ex- 흠 tract was reconstituted at $30 \mathrm{mg} / \mathrm{ml}$ in saline and examined for precipitins using Ouchterlony plates $\overparen{D}$ treated to eliminate C-substance reactions (Longbottom and Pepys, 1964). A similar extract, recon- ळ stituted at $10 \mathrm{mg} / \mathrm{ml}$, was sterilized by Millipore fil- $\overrightarrow{0}$ tration and used for skin testing.

The $A$. terreus extract produced five clearly visible $\overrightarrow{\vec{\omega}}$ precipitin lines with the patient's serum (Fig. 6). There was no reaction between the patient's serum $\overrightarrow{\vec{x}}$ and $A$. fumigatus antigen (Bencard) nor between the $A$. terreus antigen and sera which had previously. been shown to contain $A$. fumigatus antibodies. Parallel tests were conducted with the sera from 20 \& patients not known to have had contact with aspergilli using antigens from $A$. terreus and $A$. fumigatus (Bencard). No reactivity was recorded.

\section{MYCOLOGICAL METHODS (PATIENT 2)}

Specimens of sputum, urine, stool, and blood were $\frac{\mathbb{D}}{3}$ examined directly as smears processed by the methenamine silver method of Grocott (1955) and in culture on malt agar plate incubated at $26^{\circ} \mathrm{C}$ and $\vec{\theta}$ $37^{\circ} \mathrm{C}$. Necropsy materials were subject to the same $N$ schedule.

RESUlTS During the first month of a two-month sampling period the following results were recorded: Sputum A. fumigatus was cultured on six occasions the last four samples giving uncountably dense yields. Yeasts were isolated seven times, an assembly of pigmented species arising on two occasions. These were identified as Rhodotorula glutinis and $M$. pulcherrima.

A randomly distributed background flora of filamentous fungi included penicillia ( $P$. lanoso-viride, $P$. herquei, $P$. tardum, and $P$. decumbens), aspergilli ( $A$. ustus, $A$. versicolor, and $A$. niger), Ostracoderma sp., and Botrytis cinerea.

Urine $M$. pulcherrima was recovered from two samples, $R$. glutinis from two, and $A$. fumigatus from one.

Stool Candida tropicalis was the sole fungus isolated; blastospores were common in smears.

Blood All samples were negative for fungi.

During the second month of sampling $A$. fumigatus was not cultured from the sputum although on one occasion dichotomously branched hyphae were seen in smears. $A$. fumigatus occurred only twice in urine cultures. Stools gave a consistently high yield of $C$. tropicalis and smears showed numerous blastospores. At necropsy settle plates of malt agar exposed adjacent to the cadaver gave colonies of Cladosporium sp. A. fumigatus was cultured from the mycetoma and from the lower lobe of the left lung. Blood which remained in the thorax after dissection gave colonies of $M$. pulcherrima and Cladosporium sp. C. albicans and $M$. pulcherrima were isolated from the thrombus in the superior vena cava. A. fumigatus was also recovered from the cortex of the right kidney. 


\section{DISCUSSION}

The concurrence of ankylosing spondylitis and pulmonary aspergillosis may well have been fortuitous in the two patients described. Certain changes, however, may occur in the lungs in the presence of long-standing ankylosing spondylitis, and if cavitation develops there is an ideal site for colonization by fungi with airborne spores. There is a recognized association between ankylosing spondylitis and an increased incidence of pulmonary tuberculosis (Crofton and Douglas, 1969). Aspergillus species will colonize a significant proportion of residual tuberculous cavities, as has been shown by large-scale investigations (British Tuberculosis Association, 1968). Furthermore, attention has recently been drawn to a specific infiltrative and fibrosing lesion in the lungs of patients with ankylosing spondylitis, in which cyst formation occurs in the affected upper lobes (British Medical Journal, 1971). Davies (1971) has referred to a figure of over 50 reported cases. Pulmonary damage may also result from intensive midline irradiation for spinal disease.

Of the few patients in whom ankylosing spondylitis has been recorded as accompanying pulmonary mycetoma formation, that of Campbell and Clayton (1964) and that of Edwards and La Touche (1964) are noted also to have had bronchiectasis, perhaps secondary to irradiation damage. Five patients with pulmonary mycetoma and ankylosing spondylitis were reported by Krohn and Halvorsen (1968), but no details of radiotherapy were given. Leggat and de Kretser (1968) reported the development of an aspergilloma in a patient who had received radiotherapy for ankylosing spondylitis and who had fibrotic changes in both upper lobes. A mycetoma due to Allescheria boydii has been recorded in a patient with advanced ankylosing spondylitis; details of radiotherapy were not given (Adelson and Malcolm, 1968).

In a survey of patients with lung cavitation and fibrosis mimicking tuberculosis, Davies (1970) described four patients with ankylosing spondylitis having the specific upper lobe changes previously alluded to, all of whom developed unilateral or bilateral aspergillomata. One of these four was the patient reported by Leggat and de Kretser (1968). It seems probable that our own two patients are further examples of this specific pulmonary lesion in ankylosing spondylitis.

$A$. terreus is one of the most common and widely distributed of soil organisms. It is charac- terized by strongly columnar cinnamon to orange-brown conidial heads (Raper and Fennell, 1965). Although it is known to be a potential pathogen, its importance as such has previously been confined to superficial infections of the skin, nails, and external ear (English and Dalton, 1962 ; English, 1963 ; Austwick, 1965). Mahgoub, Ismail, and El Hassan (1969) have reported one proven case of human cervical lymphadenitis caused by $A$. terreus in the Sudan. In the animal sphere, it may be a cause of bovine mycotic abortion (Ainsworth and Austwick, 1959). It has also been isolated from a granulomatous skin lesion in a cow (Davis and Schaefer, 1962). Pore and Larsh (1968) have shown it to produce neurological and renal lesions in inoculated mice. Pathogenic activity of $A$. terreus in the human respiratory system has not previously been reported, although the organism has been obtained from pulmonary lesions in birds (Ainsworth and Austwick, 1955).

M. pulcherrima, a yeast previously named $C$. pulcherrima, was first isolated by Lindner in 1901. The organism is distinguishable from other yeasts by the presence of globose 'pulcherrima' cells containing a single large lipid globule (Lodder, 1970). It also elaborates a red pigment known as pulcherrimin. It is geographically widely distributed, usually being found on fruits, flowers, and other vegetable material. Ainsworth and Austwick (1959) listed the organism among a number of 'isolates of uncertain status' as a possible causative agent in mycotic mastitis in animals. There is little mention of this yeast in the literature on human disease but Lodder and Kreger-van Rij (1952) quote two instances of its isolation from human subjects-one strain from interdigital skin lesions (Pollaci and Nannizzi, 1926) and two strains from the oropharyngeal regions of normal or diseased persons (Orie, 1946).

Although the examination of the two patients described in this paper may have shed little further light on the association of bronchopulmonary aspergillosis with ankylosing spondylitis, it has brought to notice the potential pathogenicity of two fungal organisms not previously implicated in infection of the human respiratory tract. $A$. terreus was consistently isolated from the sputum of the first patient over a period of six months, and he was shown to have developed both reaginic and precipitating antibodies to this organism. The second patient had a large mycetoma caused by $A$. fumigatus, but among an assemblage of other less prominent fungal iso- 
lates was the yeast $M$. pulcherrima, which occurred in sputum and urine during life, and also in post-mortem superior vena caval thrombus. $M$. pulcherrima could not justifiably be termed a pathogen on the present evidence and was probably no more than a secondary invader, but these observations do draw attention to it as a possible infecting organism in the debilitated human subject.

The mycological studies were undertaken as part of a research programme supported by a grant from the Scottish Hospital Endowments Research Trust.

We thank Mr. E. J. C. Kerr for performing the gel diffusion tests, Mrs. M. Stewart for help with the culture and identification of the yeast species, Dr. N. MacLean for performing the necropsy on patient 2, the Medical Photography Department of the University of Edinburgh for reproduction of the chest radiographs, and Miss I. A. MaCall for secretarial assistance. We are indebted to Dr. I. W. B. Grant for encouragement and criticism and also for allowing us to study patient 1 .

\section{REFERENCES}

Adelson, H. T., and Malcolm, J. A. (1968). Endocavitary treatment of pulmonary mycetomas. Amer. Rev. resp. Dis., 98, 87.

Ainsworth, G. C., and Austwick, P. K. C. (1955). A survey of animal mycoses in Britain: general aspects. Vet. Rec., 67, 88.

_ (1959). Fungal Diseases of Animals. Commonwealth Agricultural Bureaux, Farnham Royal, Bucks.

Austwick, P. K. C. (1965). Pathogenicity. In: The Genus Aspergillus, edited by K. B. Raper and D. I. Fennell, Williams and Wilkins, Baltimore.

British Medical Journal (1971). Leading article, The lungs in ankylosing spondylitis. Brit. med. J., 3, 492.

British Tuberculosis Association (1968). Aspergillus in persistent lung cavities after tuberculosis. Tubercle (Lond.), 49, 1.

Campbell, M. J., and Clayton, Y. M. (1964). Bronchopulmonary aspergillosis. Amer. Rev. resp. Dis., 89, 186.

Crofton, J. W., and Douglas, A. C. (1969). Respiratory Diseases, 1st ed. Blackwell Scientific Publications, Oxford and Edinburgh.
Curry, J. (1949). A droplet culture method for fungus isola-듬 tion, and a staining method for diagnosis of epidermophytosis. Brit. J. Derm., 61, 54.

Davies, D. (1970). Lung fibrosis and cavitation mimicking tuberculosis. Tubercle (Lond.), 51, 246.

(1971). The lungs and ankylosing spondylitis. Brit. med. J., 3, 768.

Davis, C. L., and Schaefer, W. B. (1962). Cutaneous aspergillosis in a cow. J. Amer. vet. med. Ass., 141, 1339.

Edwards, G., and La Touche, C. J. P. (1964). The treatment of bronchopulmonary mycoses with a new antibioticpimaricin. Lancet, 1, 1349.

English, M. P. (1963). Preliminary observations on some. Aspergillus species in relation to their rôle as pathogens? of aural cavities. J. Laryng., 77, 422.

- and Dalton, G. A. (1962). An outbreak of fungal infections of post-operative aural cavities. J. Laryng., 음 76, 1.

Grocott, R. G. (1955). A stain for fungi in tissue sections and $\vec{C}$ smears. Amer. J. clin. Path., 25, 975.

Krohn, J., and Halvorsen, J. H. (1968). Aspergilloma of the lung in ankylosing spondylitis. Scand. J. resp. Dis., Suppl. 63, p. 131.

Leggat, P. O., and de Kretser, D. M. H. (1968). Aspergillus pneumonia in association with an aspergilloma. Brit. J. Dis. Chest., 62, 147.

Lindner, P. (1901). Mikroscopische Betriebskontrolle in den Gärungsgewerben, 3te Aufl. Berlin.

Lodder, J. (Ed.) (1970). The Yeasts, a Taxonomic Study, 2nd ed. North-Holland Publishing Co., Amsterdam and London.

- and Kreger-van Rij, N. J. W. (1952). The Yeasts, $a \stackrel{\mathbb{\perp}}{\circ}$ Taxonomic Study. North-Holland Publishing Co., $\overrightarrow{\vec{F}}$ Amsterdam.

Longbottom, J. L., and Pepys, J. (1964). Pulmonary aspergillosis: diagnostic and immunological significance of antigens and C-substance in Aspergillus fumigatus. J. Path. Bact., 88, 141.

Mahgoub, E. S., Ismail, S. A., and El Hassan, A. M. (1969). 뭉

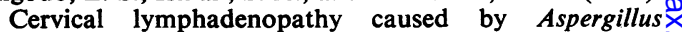
terreus. Brit. med. J., 1, 689.

Orie, N. G. M. (1946). De aanwezigheid en de beteekenis van. gisten in de luchtwegen. Diss., Utrecht. Wolter, Gronin-尺̊ gen.

Pollacci, G., and Nannizzi, A. (1926). Miceti patog. Uomo, 응 5, No. 44

Pore, R. S., and Larsh, H. W. (1968). Experimental pathology $\frac{7}{0}$ of Aspergillus terreus-flavipes group species. Sabouraudia, $6,89$.

Raper, K. B., and Fennell, D. I. (1965). The Genus Aspergillus. Williams and Wilkins, Baltimore. 